\title{
Continuous-wave biexciton lasing at room temperature using solution-processed quantum wells
}

\author{
Joel Q. Grim, Sotirios Christodoulou, Francesco Di Stasio, Roman Krahne, Roberto Cingolani, \\ Liberato Manna and Iwan Moreels*
}

Solution-processed inorganic and organic materials have been pursued for more than a decade as low-threshold, high-gain lasing media, motivated in large part by their tunable optoelectronic properties and ease of synthesis and processing.' Although both have demonstrated stimulated emission and lasing, they have not yet approached the continuouswave pumping regime ${ }^{1-8}$. Two-dimensional CdSe colloidal nanosheets combine the advantage of solution synthesis with the optoelectronic properties of epitaxial two-dimensional quantum wells. Here, we show that these colloidal quantum wells possess large exciton and biexciton binding energies of $132 \mathrm{meV}$ and $30 \mathrm{meV}$, respectively, giving rise to stimulated emission from biexcitons at room temperature. Under femtosecond pulsed excitation, close-packed thin films yield an ultralow stimulated emission threshold of $6 \mu \mathrm{J} \mathrm{cm}$, sufficient to achieve continuous-wave pumped stimulated emission, and lasing when these layers are embedded in surface-emitting microcavities.

The first demonstration of continuous-wave (c.w.) semiconductor lasing at room temperature was achieved in double heterostructure lasers over 40 years ago ${ }^{9}$. Since then, solid-state c.w. lasers have been developed at several key wavelengths, most notably a mid-infrared quantum cascade laser at $9 \mu \mathrm{m}$ (ref. 10), a $1.69 \mu \mathrm{m}$ silicon Raman laser ${ }^{11}$, and violet-blue (399 nm, ref. 12; $428 \mathrm{~nm}$, ref. 13) InGaN lasers. However, these are all fabricated via demanding microfabrication techniques, which typically require high vacuum and temperature. Solution-processed lasers, meanwhile, offer the prospect of fabrication on a wide variety of (flexible) substrates with facile, large-area methods such as spray coating or inkjet printing, but c.w. operation remains to be demonstrated before practical applications are deemed feasible.

In organic electronics, nanosecond-pumped lasing has already been achieved, yet the stimulated emission is eventually quenched by the pile-up of long-lived triplet states and concomitant singlettriplet annihilation ${ }^{7,8}$. Long-term operation is also compromised by sample degradation ${ }^{7}$, and electrically pumped lasing is still considered a significant challenge $\mathrm{e}^{7,8}$. Inorganic colloidal nanocrystals are a potential alternative material ${ }^{1,3,4}$. However, in these materials, progress was initially hampered by efficient Auger recombination, which prohibited a sustained population inversion ${ }^{3,14}$.

Auger recombination rates in nanocrystals are inversely proportional to the exciton volume and directly proportional to the electron-hole wavefunction overlap, and are therefore suppressed in nanocrystal heterostructures that allow for electron delocalization $^{15}$. As a result, stimulated emission has already been demonstrated in CdSe/CdS giant-shell colloidal quantum dots (CQdots) ${ }^{15}$ and dot-in-rods ${ }^{2}$, and in the latter, a temperature-independent stimulated emission threshold, a typical Qdot lasing characteristic, has been observed. Electron delocalization comes, however, at the expense of reduced oscillator strength of the band-edge transition. Dang and colleagues ${ }^{3}$ have shown with $\mathrm{CdSe} / \mathrm{Cd}_{0.5} \mathrm{Zn}_{0.5} \mathrm{~S}$ type I core-shell nanocrystals that an exceptionally high photoluminescence quantum efficiency of over $80 \%$ in a highly concentrated nanocrystal solution and a large Stokes shift of $\sim 20 \mathrm{~nm}$ are also sufficient conditions to reach low-threshold gain and single-exciton lasing. This achievement was probably facilitated by using nanocrystals with a graded core-shell interface, as a smoother confining potential reduces the high momentum components in the ground-state wavefunction, and this is known to reduce Auger recombination ${ }^{16,17}$.

The need for engineered heterostructures that suppress Auger recombination or achieve single-exciton gain can be circumvented altogether with two-dimensional nanocrystals, that is, colloidal quantum wells (CQwells) ${ }^{18,19}$. Here, the energy levels are quantized in only one direction, which imposes a stricter momentum conservation rule for Auger recombination compared to zerodimensional CQdots. Using CdSe CQwells with green emission, we indeed observed efficient biexciton emission at room temperature, with a lifetime consistent with strong Auger recombination suppression. This enabled us to achieve gain in CQwell thin films using only $6 \mu \mathrm{J} \mathrm{cm}^{-2}$ of excitation fluence in the pulsed regime, as well as optically pumped c.w. stimulated emission and lasing at $530 \mathrm{~nm}$.

Atomic control of the thickness of CdSe CQwells during synthesis is now possible, resulting in narrow, symmetric emission bands that can be tuned in discrete steps from blue $(460 \mathrm{~nm})$ to green $(515 \mathrm{~nm})$ and yellow $(550 \mathrm{~nm})^{20}$. Here, we optimized the synthesis of green-emitting CQwells (see Materials and Methods) to obtain samples with a typical photoluminescence quantum efficiency of $25 \%$ in solution. Figure 1a presents a transmission electron microscope (TEM) image of the CQwells. The $2.41 \mathrm{eV}$ photoluminescence peak (Fig. 1b) corresponds to a thickness of $1.5 \mathrm{~nm}$, equivalent to five CdSe monolayers (ML) according to Achtstein and co-authors $^{21}$. The lateral dimensions of $8.5 \mathrm{~nm} \times 35.3 \mathrm{~nm}$ imply that excitons only experience strong confinement in the $z$ direction. The emission and absorption spectra in Fig. 1b reveal a Stokes shift of $24 \mathrm{meV}$. Excitonic transitions associated with the heavy holes $(\mathrm{HH})$ and light holes $(\mathrm{LH})$ are also visible at $2.43 \mathrm{eV}$ and $2.57 \mathrm{eV}$, respectively. By fitting a quantum well absorption model to the spectrum (Supplementary Section 2), we determined an exciton binding energy $E_{\mathrm{b}}^{\mathrm{x}}$ of $132 \pm 20 \mathrm{meV}$, which agrees with a calculated $E_{\mathrm{b}}^{\mathrm{x}}$ of $130-175 \mathrm{meV}$ (ref. 21) for $5 \mathrm{ML}$ colloidal CdSe CQwells with lateral extensions of $20 \mathrm{~nm}$ (taking a dielectric constant of the ligands equal to 2-3). The high value is consistent with other Qwell systems ${ }^{22}$ and can be correlated with the 
a
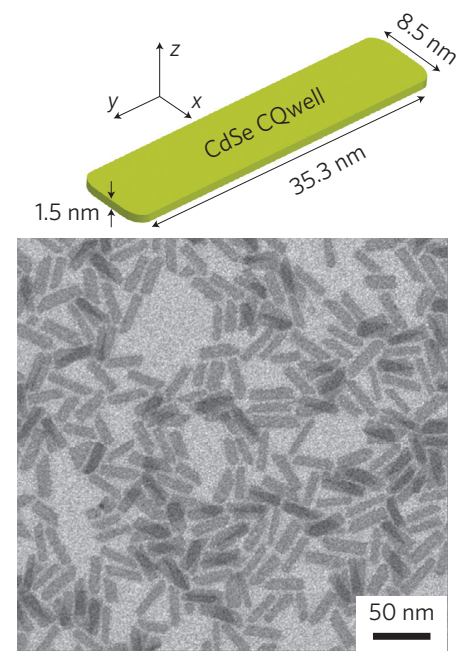

b

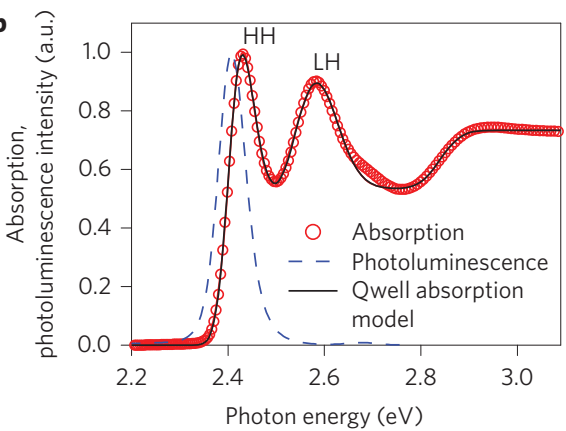

Figure 1 | Structural and optical CQwell properties. a, TEM image of CdSe CQwells (lateral dimensions $8.5 \mathrm{~nm} \times 35.3 \mathrm{~nm}$ ) and sketch of a CQwell, indicating a thickness of $1.5 \mathrm{~nm}$ (ref. 21). b. Absorption (circles) and photoluminescence (dashed line) spectra of the CdSe CQwells used in the study. The two peaks correspond to the excitonic transitions associated with the heavy holes $(\mathrm{HH})$ and light holes $(\mathrm{LH})$. Absorption data are fit with a quantum well absorption model (full line), yielding an exciton binding energy of $132 \mathrm{meV}$.

exceptionally strong confinement of the two-dimensional exciton in the $1.5 \mathrm{~nm}$ thin Qwell, further enhanced by the large dielectric mismatch between the inorganic CdSe CQwells and the organic environment. As we exceed the expected fourfold enhancement for two-dimensional-confined excitons (bulk CdSe $\left.E_{\mathrm{b}}^{\mathrm{x}}=15 \mathrm{meV}\right)^{23}$, excitons may still experience weak confinement in the lateral dimensions, either due to the finite CQwell size or to small potential well fluctuations.

Detailed measurements of the biexciton emission and associated Auger recombination were performed on close-packed thin films that were obtained by dropcasting the CQwells from toluene onto a sapphire substrate. The top and bottom panels in Fig. 2a show a spectrally symmetric photoluminescence from $F_{0}=0.4 \mu \mathrm{J} \mathrm{cm}^{-2}$ excitation, and a broadened spectrum for $F_{0}=32 \mu \mathrm{J} \mathrm{cm}^{-2}$. The spectra in Fig. $2 \mathrm{~b}$ were obtained by temporally integrating the streak images in Fig. 2a over the first nanosecond. They are fit by a pseudo-Voigt function with line shape $V_{\mathrm{x}}$ for the low-fluence spectrum (Supplementary Section 4) and a sum of two Voigt functions $V=A_{\mathrm{x}} V_{\mathrm{x}}+A_{\mathrm{xx}} V_{\mathrm{xx}}$, where $V_{\mathrm{xx}}$ represents the second emission peak, with the same line shape as $V_{\mathrm{x}}$, only shifted to lower energy. The respective amplitudes $A_{\mathrm{x}}$ and $A_{\mathrm{xx}}$ show a linear $\left(I \propto I_{0}\right)$ and quadratic $\left(I \propto I_{0}^{2.2}\right)$ dependence on the excitation fluence, respectively. The latter confirms that the red band is due to a biexciton transition (Fig. 2c). The energy difference between the peak positions yields a biexciton binding energy of about $E_{\mathrm{b}}^{\mathrm{xx}}=30 \mathrm{meV}$. Theoretical predictions find that the two-dimensional heavy-hole biexciton and exciton have a ratio of $E_{\mathrm{b}}^{\mathrm{xx}} / E_{\mathrm{b}}^{\mathrm{x}}=0.228$ (ref. 24). Using $E_{\mathrm{b}}^{\mathrm{X}}=132 \pm 20 \mathrm{meV}$, we calculate $E_{\mathrm{b}}^{\mathrm{Xx}}=30 \pm 5 \mathrm{meV}$, which is remarkably close to the experimental value. A calculation using the CdSe bulk $E_{\mathrm{b}}^{\mathrm{x}}$ of $15 \mathrm{meV}$ predicts a two-dimensional binding energy of $14 \mathrm{meV}$. Similar enhancements compared to the ideal two-dimensional case were observed in self-assembled CdSe Qdots formed by Cd-rich regions inside a CdZnSe Qwell, where $E_{\mathrm{b}}^{\mathrm{xx}}$ was equal to $19-26 \mathrm{meV}$ (ref. 25) and $38 \mathrm{meV}$ (ref. 26), respectively. The biexciton binding energy again suggests a residual in-plane centre-of-mass confinement, yet, more importantly, our data predict that this is beneficial in our case, as biexcitons in CdSe CQwells are stable at room temperature.

CdSe CQwells have a band-edge transition with so-called giant oscillator strength, where a coherent phasing of dipoles over many unit cells results in a dipole matrix element much larger than can be achieved by a single atom ${ }^{27}$. This is manifested by the fast single-exciton photoluminescence decay kinetics observed in Fig. 2d. Fitting a multi-exponential function to the spectrally integrated exciton decay yields an effective lifetime $\tau_{\mathrm{x}}=438 \mathrm{ps}$, together with a lower intensity tail with a 5.1 ns decay constant. The photoluminescence trace at higher excitation fluence yields a slightly faster decay time of $\tau_{2}=296 \mathrm{ps}$, with a $1.4 \mathrm{~ns}$ tail. Focusing on the picosecond decay times and using the respective areas of the exciton and biexciton emission from Fig. 2b, we can estimate a biexciton lifetime of $\tau_{\mathrm{xx}}=\left(\tau_{2}-A_{\mathrm{x}} \tau_{\mathrm{x}}\right) / \mathrm{A}_{\mathrm{xx}}=124$ ps. The $\tau_{\mathrm{xx}} / \tau_{\mathrm{x}}$ ratio of $1: 3.5$ is similar to epitaxial systems based on CdSe (ref. 26) or GaAs (ref. 28) and, considering that typical $\tau_{\mathrm{xx}} / \tau_{\mathrm{x}}$ decay time ratios for type I CQdots are close to 1:100 (ref. 3), it excludes the possibility that the biexciton lifetime is dominated by nonradiative Auger recombination. This is supported by a recent study that finds Auger lifetimes up to 10 ns (ref. 29), almost two orders of magnitude larger than the measured radiative lifetimes.

Stimulated emission was first investigated using femtosecond pulsed excitation. Recent literature results have demonstrated that stimulated emission ${ }^{5}$ and lasing ${ }^{6}$ are feasible under these conditions. Figure 3a shows a streak camera image of the stimulated emission collected at $90^{\circ}$ from a focused stripe (Supplementary Section 5). The characteristic band narrowing results in a $6 \mathrm{~nm}$ stimulated emission peak width. The ultrafast decay time falls within the 10 ps response time of the streak camera, and unambiguously assigns the peak to stimulated emission. It is also evident from Fig. 3a and the intensity-dependent spectra in Fig. 3b that the stimulated emission originates at the energy position of the biexciton transition. The integrated stimulated emission intensity shows a clear threshold behaviour, with the biexciton photoluminescence becoming stimulated emission at an ultralow threshold of $6 \mu \mathrm{J} \mathrm{cm}^{-2}$ (Supplementary Section 5), about 25 times smaller than $\mathrm{CdSe} / \mathrm{Cd}_{0.5} \mathrm{Zn}_{0.5} \mathrm{~S}$ core-shell ${ }^{3}$ and $\mathrm{CdSe} / \mathrm{CdS}$ dot-in-rods ${ }^{2}$ with similar emission wavelengths.

We have therefore obtained a material with several unique properties that are key to low-threshold lasing. Compared to zerodimensional CQdots, CQwells have a larger oscillator strength and therefore larger spontaneous and stimulated emission crosssections. Due to the (nearly) atomically flat CQwells, almost all nanocrystals can contribute to population inversion, and losses due to sample heterogeneity are largely avoided. This is also demonstrated by photoluminescence excitation (PLE) spectroscopy, where both the band-edge absorbance and the PLE peak have similar linewidths of $34.8 \pm 0.5 \mathrm{meV}$ and $33.8 \pm 0.4 \mathrm{meV}$, respectively (Supplementary Section 6). The large biexciton binding energy also results in strongly redshifted biexciton emission, further circumventing linear absorption losses. In this context, the absence of Auger recombination implies that nonlinear nonradiative losses are also minimized. Finally, even at room temperature, the gain can arise from a biexciton population, whereas in other QWell systems gain is typically obtained from electron-hole plasma at 

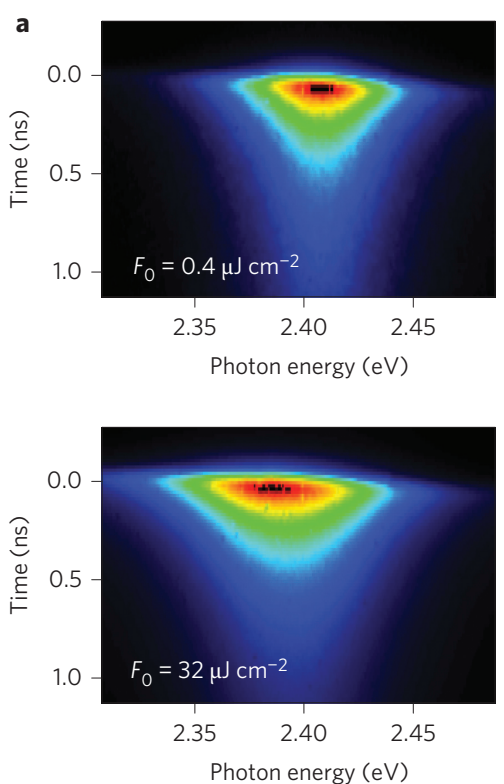
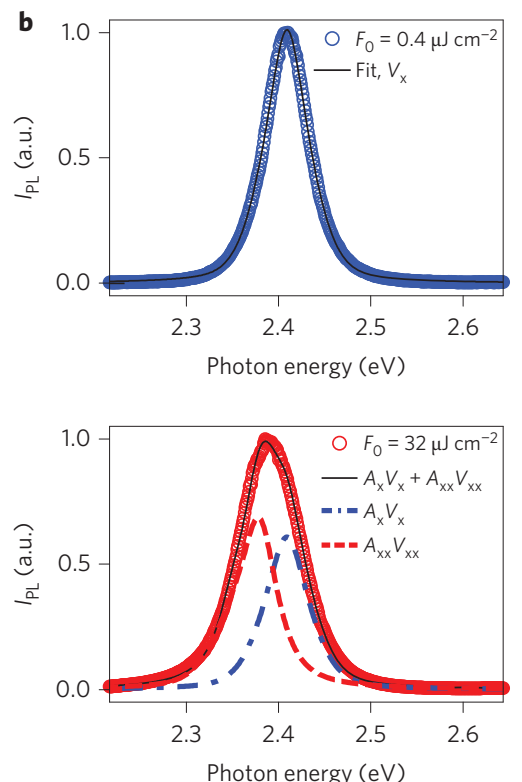
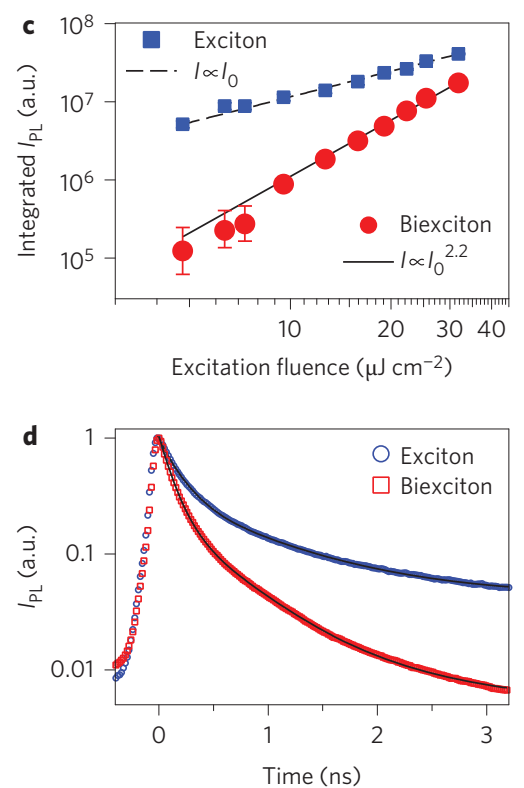

Figure 2 | Power-dependent and time-resolved photoluminescence data. a, Streak camera images of CdSe CQwells excited with $1 \mathrm{kHz}$, $70 \mathrm{fs}$ laser pulses, showing low-fluence (top) and high-fluence (bottom) emission. $\mathbf{b}$, The time-integrated spectrum (over the first nanosecond) for a fluence of $0.4 \mu \mathrm{J} \mathrm{cm}^{-2}$ shows a single symmetric peak. At a fluence of $32 \mu \mathrm{J} \mathrm{cm}^{-2}$, the spectrum contains two components, $A_{x} V_{x}$ and $A_{x x} V_{x x}$. c, The integrated intensities confirm that the redshifted peak is due to biexciton emission. d, Spectrally integrated decay traces, from which exciton and biexciton lifetimes of 438 ps and 124 ps, respectively, can be deduced.
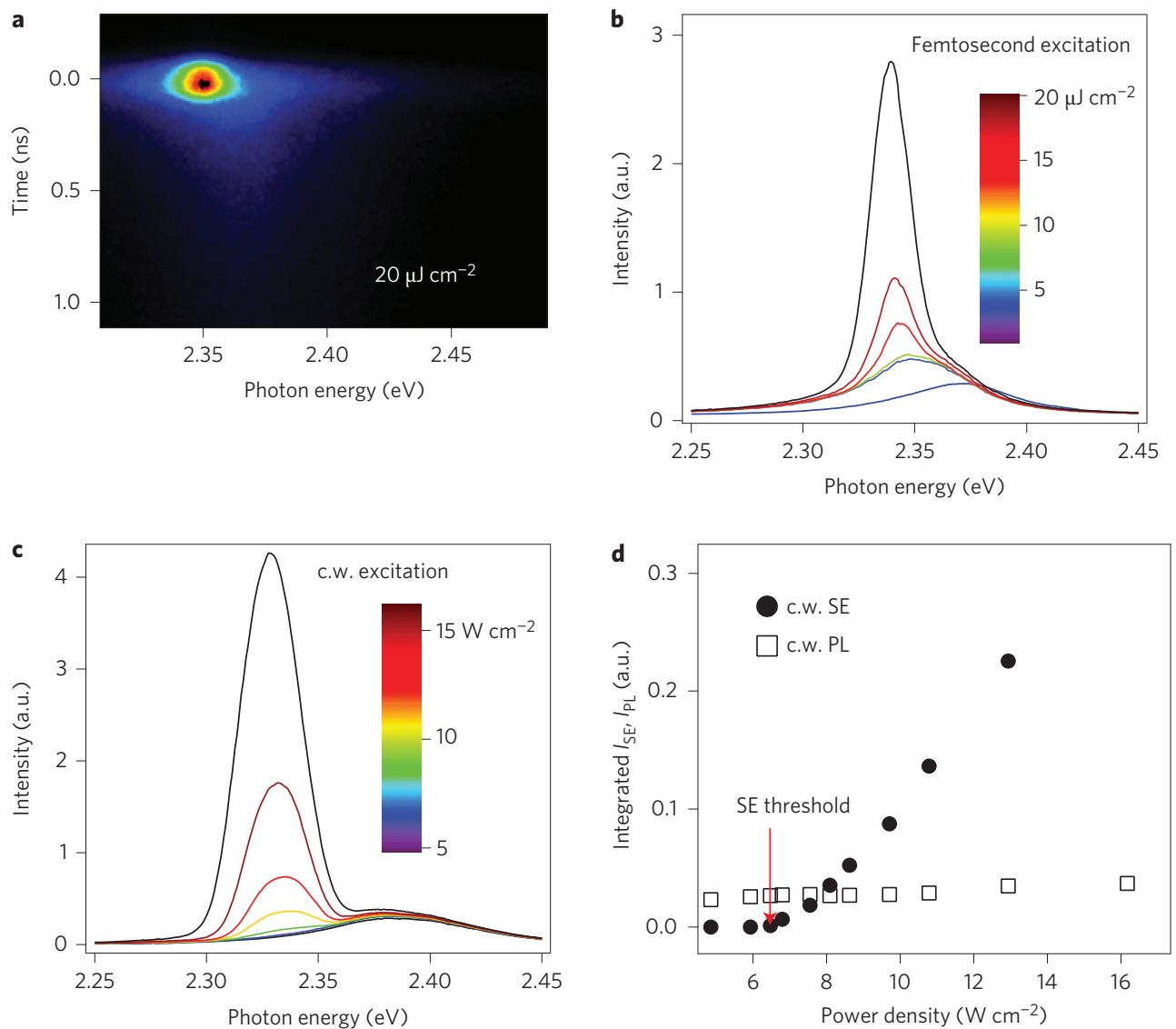

Figure 3 | Stimulated emission under femtosecond and c.w. pumped conditions. a, Streak camera image showing fast, narrow-band stimulated emission under femtosecond excitation. The spectral and temporal ranges are the same as in Fig. 2a to assist visual comparison. $\mathbf{b}$, Excitation fluence dependence of stimulated emission under femtosecond excitation. The low-intensity single-exciton photoluminescence first develops into biexciton emission, followed by gain narrowing and stimulated emission at even higher fluences. c, Similar spectra are obtained under c.W. excitation, yet without the observation of a clear biexciton photoluminescence peak. $\mathbf{d}$, Intensity dependence for c.w. excitation shows a threshold at $6.5 \mathrm{~W} \mathrm{~cm}^{-2}$ for the stimulated emission (SE) peak and saturation behaviour for the residual photoluminescence $(\mathrm{PL})$. 
a

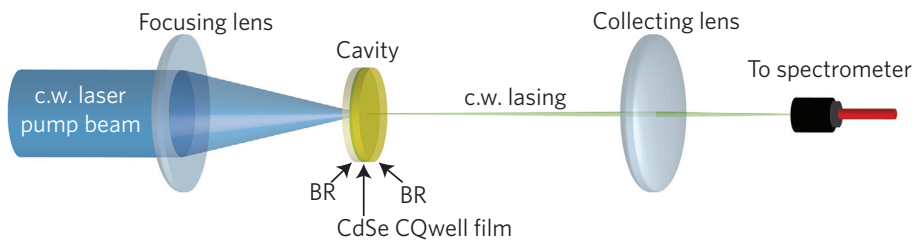

b 0.0

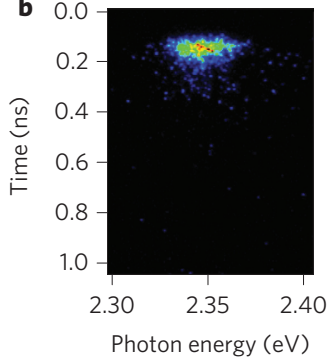

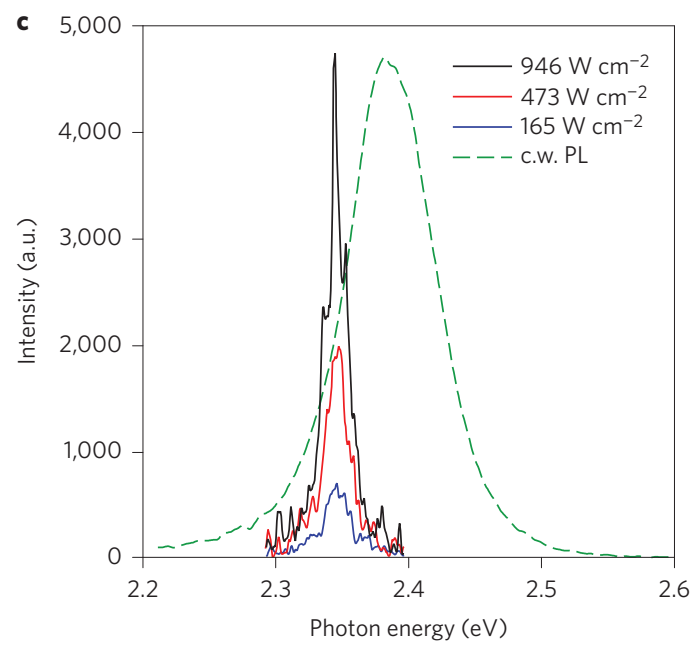

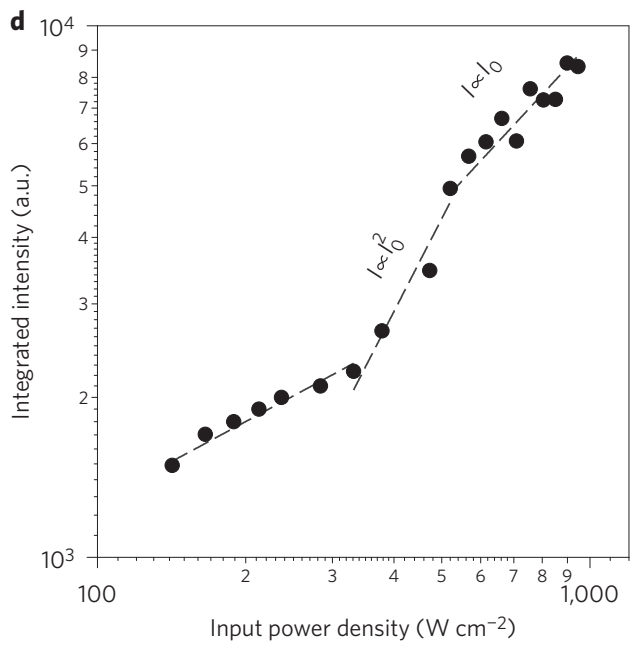

Figure 4 | Continuous-wave lasing in a CQwell-based microcavity at room temperature. a, Schematic of the laser cavity with identical Bragg reflectors (BRs) with $99.5 \%$ reflectivity at $530 \mathrm{~nm}$. b. Pulsed femtosecond measurements produce the same lasing peak as with c.w. pumping and confirm the expected ultrafast response of the emission peak. c, Rise of the c.w. pumped lasing peak centred at $2.34 \mathrm{eV}$ (530 nm) with a narrow central mode visible. The photoluminescence ( $\mathrm{PL}$, both exciton and biexciton emission) spectrum of a thin film is shown for comparison. $\mathbf{d}$, The integrated intensity of the 2.34 eV peak has an S-shaped power dependence, with a superlinear transition phase around a threshold of $440 \mathrm{~W} \mathrm{~cm}^{-2}$ giving way to a linear response when the lasing threshold is surpassed.

these temperatures ${ }^{30}$. The four-level system obtained here represents a more beneficial scheme to achieve low-threshold lasing ${ }^{30}$.

When switching to c.w.-pumped excitation $(\lambda=444 \mathrm{~nm})$, a stimulated emission peak is clearly observed with spectral position and linenarrowing (Fig. 3d) consistent with that seen for the femtosecond excitation. We find a c.w. stimulated emission threshold of $6.5 \mathrm{~W}$ $\mathrm{cm}^{-2}$, a value that can be reached using low-power c.w. excitation sources such as blue-light-emitting or laser diodes. Using the variable stripe length (VSL) method ${ }^{31}$, we determined modal gain values of $g_{\mathrm{cw}}=121 \pm 1 \mathrm{~cm}^{-1}$ and $g_{\mathrm{fs}}=521 \pm 50 \mathrm{~cm}^{-1}$ for the CQwell thin films (Supplementary Section 5). The lower $g_{\mathrm{cw}}$ compared to $g_{\mathrm{fs}}$ is the result of a smaller sustained biexciton population under c.w. conditions, confirmed by the absence of clear biexciton emission before stimulated emission onset in Fig. 3c. This is probably due to enhanced nonradiative Auger recombination and exciton-biexciton dissociation kinetics when compared to femtosecond excitation. However, even under these conditions, $g_{\mathrm{cw}}$ is comparable to femtosecond-excited gain in CQdots ${ }^{1,3}$ and typical gain values in organic materials ${ }^{32}$.

As a proof of concept, in Fig. 4 we demonstrate c.w. pumped lasing by enclosing a $40 \mu \mathrm{m}$ dropcast thin film of CdSe CQwells in an optical cavity constructed from two Bragg reflectors (Supplementary Sections 7 and 8). The Bragg reflectors have a reflectivity of $99.5 \%$ at the $530 \mathrm{~nm}$ emission peak of the CQwells and transmit $90 \%$ of the $405 \mathrm{~nm}$ c.w. and femtosecond excitation. As for stimulated emission, we measured a quasi-instantaneous response of an overall $2.9 \mathrm{~nm}$ (full-width at half-maximum, FWHM) multimode lasing peak in the femtosecond-pulsed excitation mode (Fig. 4b, Supplementary Fig. 10). Under c.w. pumping conditions, we observe a similar behaviour (Fig. 4c,d,
Supplementary Fig. 9), with a power-dependent emission displaying an S-shaped curve that is a characteristic signature of the cavity reaching laser action. Indeed, below threshold, the photoluminescence grows sublinearly, followed by a transition region with a superlinear increase in stimulated emission (power slope of about 2 ), and finally a linear regime when we clearly obtain lasing (Fig. 4d). The threshold of $18.6 \mathrm{~mW}$, corresponding to a power density of $440 \mathrm{~W} \mathrm{~cm}^{-2}$, again places the required excitation density well within the range of existing blue-light sources. Due to the $40 \mu \mathrm{m}$ cavity length, the observed lasing peak is an envelope, yet with a dominant mode with $0.85 \mathrm{~nm}$ FWHM clearly visible at high pumping power (Fig. 4c). The device stability was tested with $1 \mathrm{~h}$ of continuous $500 \mathrm{~W} \mathrm{~cm}^{-2}$ c.w. excitation (a fluence that is above the lasing threshold shown in Fig. 4 d), with a resulting $50 \%$ reduction in lasing output, which is favourable compared to similar devices ${ }^{3}$. An excitation density of $800 \mathrm{~W} \mathrm{~cm}^{-2}$ leads to a $90 \%$ reduction in the same timeframe; however, further development could improve the performance, for example through the addition of a heat sink or the synthesis of core-shell CQwells.

In conclusion, we have shown that colloidal CdSe quantum wells share many features with epitaxial systems, making them suitable for use as high-gain materials. The infinite potential barriers provided by the low-dielectric-constant environment, combined with detailed control over the lateral sizes during colloidal synthesis, provide an exceptionally high (bi-)exciton binding energy and therefore a unique opportunity to investigate the dynamics of two-dimensional-confined excitons and biexcitons at room temperature. The suspended QWells are easily processed by wet chemical methods. Here, we have obtained c.w. pumped lasing at $530 \mathrm{~nm}$ using a simple dropcast gain layer. This leaves ample room for 
sample and device optimization, for example in the form of coreshell CQwells ${ }^{33}$ and in the direction of single-mode cavities, respectively. Considering the extended lateral dimensions, CQwell systems might even lead towards solution-processed electrically injected lasers. We thus foresee that these materials may finally provide a viable alternative to existing laser gain media.

\section{Materials and methods}

Synthesis of CdSe colloidal quantum wells. Cd-myristate (170 mg), Se (24 mg) and octadecene $(15 \mathrm{ml})$ were introduced into a three-neck flask and degassed under vacuum for $30 \mathrm{~min}$ at $100^{\circ} \mathrm{C}$. Under an argon atmosphere, the temperature was increased to $210^{\circ} \mathrm{C}$, and when this temperature was reached, $90 \mathrm{mg}$ hydrated Cd-acetate $\left(\mathrm{Cd}(\mathrm{Ac})_{2} \cdot 4 \mathrm{H}_{2} \mathrm{O}\right)$ was added swiftly. The mixture was further heated to $240{ }^{\circ} \mathrm{C}$ and kept at this temperature for $10 \mathrm{~min}$. The CQwell solution also contained a fraction of spherical clusters, which were separated from the CQwells by selective precipitation.

Steady-state photoluminescence spectroscopy. Steady-state photoluminescence, PLE spectra and photoluminescence quantum efficiency measurements were performed with an Edinburgh Instruments FLS920 spectrofluorometer. Samples were excited with a $450 \mathrm{~W}$ xenon lamp. The CdSe CQwells were dispersed in $3 \mathrm{ml}$ toluene with an optical density of 0.1 at $400 \mathrm{~nm}$ using a quartz cuvette with an optical path of $10 \mathrm{~mm}$. This wavelength was also used for the photoluminescence quantum efficiency measurements.

Ultrafast optical spectroscopy. Time-resolved photoluminescence measurements with picosecond time resolution were performed with a Hamamatsu Photonics streak camera. Samples were dropcast on a sapphire substrate for both room- and low-temperature measurements. They were excited with a $405 \mathrm{~nm}, 70 \mathrm{fs}$ amplified Ti:sapphire laser with a repetition rate of $1 \mathrm{kHz}$, or a $50 \mathrm{~mW} 444 \mathrm{~nm}$ c.w. laser source for the VSL measurements and a $110 \mathrm{~mW} 405$ c.w. laser to excite the Bragg reflector cavity. The latter ensured a higher possible excitation fluence due to the larger CdSe CQwell absorption cross-section at $405 \mathrm{~nm}$ and higher maximal laser power. For the photoluminescence spectroscopy at $4 \mathrm{~K}$, samples were cooled with a closed-cycle helium cryostat (Advanced Research Systems).

Received 12 March 2014; accepted 26 August 2014; published online 5 October 2014

\section{References}

1. Klimov, V. I. Optical gain and stimulated emission in nanocrystal quantum dots. Science 290, 314-317 (2000).

2. Moreels, I. et al. Nearly temperature-independent threshold for amplified spontaneous emission in colloidal CdSe/CdS quantum dot-in-rods. Adv. Mater. 24, OP231-OP235 (2012).

3. Dang, C. et al. Red, green and blue lasing enabled by single-exciton gain in colloidal quantum dot films. Nature Nanotech. 7, 335-339 (2012).

4. Grivas, C. et al. Single-mode tunable laser emission in the single-exciton regime from colloidal nanocrystals. Nature Commun. 4, 2376 (2013).

5. She, C. et al. Low-threshold stimulated emission using colloidal quantum wells. Nano Lett. 14, 2772-2777 (2014).

6. Guzelturk, B., Kelestemur, Y., Olutas, M., Delikanli, S. \& Demir, H. V. Amplified spontaneous emission and lasing in colloidal nanoplatelets. ACS Nano 8, 6599-6605 (2014).

7. Grivas, C. \& Pollnau, M. Organic solid-state integrated amplifiers and lasers. Laser Photon. Rev. 6, 419-462 (2012).

8. Clark, J. \& Lanzani, G. Organic photonics for communications. Nature Photon. 4, 438-446 (2010).

9. Alferov, Z. I. et al. Investigation of the influence of the AlAs-GaAs heterostructure parameters on the laser threshold current and the realization of continuous emission at room temperature. Sov. Phys. Semicond. 4, 1573-1575 (1971).

10. Beck, M. et al. Continuous wave operation of a mid-infrared semiconductor laser at room temperature. Science 295, 301-305 (2002).

11. Rong, H. et al. A continuous-wave Raman silicon laser. Nature 433, 725-728 (2005).

12. Someya, T. Room temperature lasing at blue wavelengths in gallium nitride microcavities. Science 285, 1905-1906 (1999).

13. Tamboli, A. C. et al. Room-temperature continuous-wave lasing in GaN/InGaN microdisks. Nature Photon. 1, 61-64 (2007).
14. Klimov, V. I. Quantization of multiparticle auger rates in semiconductor quantum dots. Science 287, 1011-1013 (2000).

15. García-Santamaría, F. et al. Suppressed auger recombination in 'giant' nanocrystals boosts optical gain performance. Nano Lett. 9, 3482-3488 (2009)

16. Climente, J. I., Movilla, J. L. \& Planelles, J. Auger recombination suppression in nanocrystals with asymmetric electron-hole confinement. Small 8, 754-759 (2012).

17. Bae, W. K. et al. Controlled alloying of the core-shell interface in CdSe/CdS quantum dots for suppression of Auger recombination. ACS Nano 7, 3411-3419 (2013).

18. Joo, J., Son, J. S., Kwon, S. G., Yu, J. H. \& Hyeon, T. Low-temperature solutionphase synthesis of quantum well structured CdSe nanoribbons. J. Am. Chem. Soc. 128, 5632-5633 (2006).

19. Ithurria, S. \& Dubertret, B. Quasi 2D colloidal CdSe platelets with thicknesses controlled at the atomic level. J. Am. Chem. Soc. 130, 16504-16505 (2008)

20. Ithurria, S. et al. Colloidal nanoplatelets with two-dimensional electronic structure. Nature Mater. 10, 936-941 (2011).

21. Achtstein, A. W. et al. Electronic structure and exciton-phonon interaction in two-dimensional colloidal CdSe nanosheets. Nano Lett. 12, 3151-3157 (2012).

22. Sun, H. D. et al. Enhancement of exciton binding energies in $\mathrm{ZnO} / \mathrm{ZnMgO}$ multiquantum wells. J. Appl. Phys. 91, 1993 (2002).

23. Voigt, J., Spiegelberg, F. \& Senoner, M. Band parameters of CdS and CdSe single crystals determined from optical exciton spectra. Phys. Status Solidi 91, 189-199 (1979).

24. Birkedal, D., Singh, J., Lyssenko, V., Erland, J. \& Hvam, J. Binding of quasi-twodimensional biexcitons. Phys. Rev. Lett. 76, 672-675 (1996).

25. Patton, B., Langbein, W. \& Woggon, U. Trion, biexciton, and exciton dynamics in single self-assembled CdSe quantum dots. Phys. Rev. B 68, 125316 (2003).

26. Woggon, U. et al. Huge binding energy of localized biexcitons in CdS/ZnS quantum structures. Phys. Rev. B 61, 12632-12635 (2000).

27. Feldmann, J. et al. Linewidth dependence of radiative exciton lifetimes in quantum wells. Phys. Rev. Lett. 59, 2337-2340 (1987).

28. Kim, J., Wake, D. \& Wolfe, J. Thermodynamics of biexcitons in a GaAs quantum well. Phys. Rev. B 50, 15099-15107 (1994).

29. Kunneman, L. T. et al. Bimolecular auger recombination of electron-hole pairs in two-dimensional CdSe and CdSe/CdZnS core/shell nanoplatelets. J. Phys. Chem. Lett. 4, 3574-3578 (2013).

30. Homburg, O. et al. Biexcitonic gain characteristics in ZnSe-based lasers with binary wells. Phys. Rev. B 60, 5743-5750 (1999).

31. Malko, A. V. et al. From amplified spontaneous emission to microring lasing using nanocrystal quantum dot solids. Appl. Phys. Lett. 81, 1303-1305 (2002).

32. Chénais, S. \& Forget, S. Recent advances in solid-state organic lasers. Polym. Int. 61, 390-406 (2012).

33. Mahler, B., Nadal, B., Bouet, C., Patriarche, G. \& Dubertret, B. Core/shell colloidal semiconductor nanoplatelets. J. Am. Chem. Soc. 134, 18591-18598 (2012).

\section{Acknowledgements}

The research leading to these results has received funding from the European Union's Seventh Framework Program (FP7/2007-2013, under grant agreement no. 298022, NIRPLANA) and the CARIPLO Foundation (NANOCRYSLAS). M. Scotto is thanked for valuable technical assistance.

\section{Author contributions}

J.Q.G., F.D.S. and I.M. conceived the experiments. S.C. synthesized the CdSe CQwells and measured the optical properties of the CQwells in solution. J.Q.G. and I.M. performed the ultrafast optical spectroscopy and lasing experiments. J.Q.G., S.C. and F.D.S. fabricated the microcavity devices, with F.D.S and R.K. performing structural characterization. J.Q.G., R.C., L.M. and I.M. analysed and interpreted the data. J.Q.G. and I.M wrote the manuscript, with contributions from all authors.

\section{Additional information}

Supplementary information is available in the online version of the paper. Reprints and permissions information is available online at www.nature.com/reprints. Correspondence and requests for materials should be addressed to I.M.

\section{Competing financial interests}

The authors declare no competing financial interests. 